\title{
Modélisation de l'évaporation globale d'un couvert forestier I - Principes physiques et description du modèle
}

\author{
P. CHASSAGNEUX, E. CHOISNEL \\ Météorologie Nationale, Subdivision d'Agrométéorologie \\ 2, avenue Rapp, F 75007 Paris
}

\begin{abstract}
Résumé
Cet article présente un modèle physique de simulation de l'évaporation globale et du bilan hydrique de deux types de couvert forestier, l'un de hêtre (Fagus silvatica L.), l'autre de Douglas (Pseudotsuga Menziesii). Il utilise des mesures météorologiques de routine couramment disponibles. Dans cette première partie sont détaillées les équations régissant le calcul de chacun des termes de ces bilans ainsi que l'estimation des paramètres du modèle liés à la structure du couvert et à son écophysiologie, notamment l'indice foliaire. L'analyse fine du processus physique d'interception justifie le choix du pas de temps horaire. Deux schémas de résistances en série sont utilisés respectivement pour la régulation de la réévaporation de l'eau interceptée et de la transpiration des arbres.

La gestion de l’eau dans le sol est réalisée par un modèle à double réservoir. Pour le couvert de hêtre la prise en compte de la phénologie, par le biais de l'évolution de l'indice foliaire, apparaît être un élément essentiel intervenant spécifiquement à différents niveaux du modèle.
\end{abstract} modèle.

Mots clés: Bilan hydrique, évaporation, indice foliaire, interception, météorologie,

\section{Introduction}

Les processus d'évaporation au-dessus et au sein d'un couvert forestier sont évalués le plus souvent de façon indirecte, et pour des intervalles de temps de l'ordre de quelques jours, par des mesures d'humidité du sol sous couvert forestier (AusSENAC \& Granier, 1979). Ces échanges sont réglés par un déterminisme énergétique lui-même fonction des variables météorologiques caractéristiques de l'état de l'atmosphère et des échanges d'énergie et d'eau à l'interface sol-atmosphère.

Or l'évaporation globale d'un couvert forestier est la somme de l'évapotranspiration réelle de l'arbre et de la réévaporation par intermittence de l'eau de pluie interceptée par le feuillage pendant et immédiatement après un épisode pluvieux. Du point de vue micrométéorologique ces processus doivent être analysés à un faible pas de temps, de l'ordre de l'heure ou de la demi-heure si l'on veut serrer de près la réalité physique. La présente étude, relative au climat à tendance continentale de l'Est de la France et à deux espèces forestières communes dans ces régions : Fagus silvatica L. (hêtre) et Pseudotsuga menziesii (Douglas), a pour but d'élaborer une 
modélisation à pas de temps fin (heure) permettant d'estimer les échanges de masse d'eau et d'énergie d'une parcelle forestière, en combinant variables météorologiques et paramètres écophysiologiques essentiels : l'interception de la pluie d'une part, la transpiration de l'arbre d'autre part.

L'interception a déjà fait l'objet de mesures précises (Aussenac \& Boulangeat, 1980 ) et de modélisations fines des transferts d'eau au niveau du feuillage (RuTTER et al., 1971). Les bilans de masse et d'énergie calculés à partir de variables météorologiques ont été étudiés pour le Douglas (Avery \& Fritschen, 1971).

La présente étude prend en compte pour un couvert de hêtre l'évolution des paramètres de la croissance (indice foliaire) et les phases de développement au printemps.

Cette première partie de l'article ne concerne que la description du modèle. Les résultats font l'objet de la seconde partie de l'article.

\section{Données expérimentales utilisées}

Toutes les données expérimentales utilisées pour le calibrage du modèle proviennent du Centre National de Recherches Forestières à Nancy (Station de Sylviculture et de Production) dans la forêt d'Amance (1 250 ha). Deux types de mesures expérimentales ont servi à calibrer et tester ce modèle :

- des mesures d'interception dans les peuplements (pour la hêtraic en 1966-1967 et pour le Douglas en 1977-1978) (Aussenac, 1968 ; Aussenac \& Boulangeat, 1980);

- des mesures hebdomadaires de profils d'humidité volumique réalisés pat* sondage neutronique ( 5 années de mesures en hêtraie, 2 années en Douglas). La parcelle de hêtraie a une superficie de $1000 \mathrm{~m}^{2}$ environ, avec une densité de 744 arbres à l'hectare (AUSSENAC \& GRANIER, 1979). Les profils ont été moyennés sur 12 tubes individuels. La parcelle de Douglas a une superficie de $990 \mathrm{~m}^{2}$ environ, avec une densité de 2229 arbres à l'hectare. Les profils ont été moyennés sur 6 tubes individuels.

\section{Hypothèses de départ}

La conception de ce modèle repose sur un certain nombre d'hypothèses qu'il semble important d'énoncer :

- le stockage de chaleur dans l'arbre et dans l'air au sein du couvert végétal est supposé négligeable (Choisnel, $1985 \mathrm{a}$ );

- une température de surface du couvert est définie: elle sert à évaluer les transferts d'énergie, d'une part par rayonnement propre du couvert vers l'atmosphère $\left(\varepsilon \sigma \mathrm{T}^{+}\right)$et d'autre part par échanges convectifs. Cette variable est interne au modèle et ne peut être directement comparéc à une mesure ;

- on suppose qu'il n'y a pas de variation du stock d'eau dans l'arbre;

- la régulation de l'évapotranspiration réelle est double : 
- en fonction de la disponibilité de l'eau dans le sol,

- en fonction des conditions météorologiques (qui jouent notamment sur la résistance stomatique);

- la variable $S$ (capacité maximale de saturation) est définie comme étant la quantité d'eau interceptée par le feuillage, au-delà de laquelle il y a égouttement en atmosphère calme (AuSSENAC, 1975);

- le rapport $\mathrm{C} / \mathrm{S}$ de la quantité d'eau interceptée au niveau du feuillage à la capacité maximale de saturation est pris comme variable indicatrice du pourcentage de surface non-transpirante (hypothèse de Rutrer également) de la façon suivante :

Si la forêt est totalement mouillée, l'évaporation globale ET est uniquement due à l'eau interceptée $(C \geqslant S)$ alors :

$$
\mathrm{ET}=\mathrm{E}_{\mathrm{max}}
$$

Dans le cas opposé (feuilles sèches) l'évaporation globale se réduit à la transpiration $(\mathrm{C}=0)$.

$$
\mathrm{ET}=\mathrm{ETR}
$$

Dans le cas général la partition entre ces deux niveaux différents est fonction linéaire de $\mathrm{C} / \mathrm{S}$ (avec $\mathrm{C}<\mathrm{S}$ ).

$$
E T=\left(1-\frac{C}{S}\right) E T R+\frac{C}{S} E_{\text {max }}
$$

Enfin le pas de temps du modèle est l'heure.

\section{Les équations du modèle}

Le modèle présenté ici consiste à gérer deux réservoirs : le réservoir d'eau dans le sol et le réservoir d'eau interceptée. Pour ce faire quatre bilans successifs sont effectués :

- un bilan radiatif (calcul du rayonnement net du couvert forestier);

— un bilan d'énergie de surface du couvert (calcul de ET);

- deux bilans hydriques (évolution de la réserve en eau du sol et du stock d'eau interceptée).

Le bilan de l'eau du sol a été effectué suivant le modèle de sol à double-réservoir de Choisnel (1985 b). Ce système gère un réservoir superficiel alimenté au printemps et en été de façon intermittente par les pluies, et un réservoir profond qui n'est réalimenté qu'en période de drainage, principalement en hiver. La gestion de ces deux réservoirs analogiques permet de réguler l'évapotranspiration en fonction de la profondeur à laquelle se trouve l'eau dans le réservoir superficiel ou, s'il est vide, dans le réservoir profond.

\subsection{Gestion de l'eau interceptée}

Ce réservoir est géré heure par heure. Une lame d'eau de pluie est réestimée pour chaque tranche horaire à partir du code «temps présent» (cf. 4.2.). Une fraction $p$ 
de cette précipitation atteint directement le sol sans toucher les arbres. Le feuillage reçoit donc $(1-p) P_{i}$. Il perd $E_{i}$ par évaporation et $D$ par égouttement (fig. 1).

On a :

$$
\frac{\Delta \mathrm{C}}{\Delta \mathrm{t}}=(1-\mathrm{p}) \mathrm{P}_{\mathrm{j}}-\mathrm{E}_{\mathrm{i}}-\mathrm{D}\left(\mathrm{en} \mathrm{mm} \cdot \mathrm{h}^{-1}\right)
$$

où C représente la quantité d'eau présente sur le feuillage et $\mathrm{P}_{1}$ la précipitation incidente.

L'égouttement ne se produit que si $\mathrm{C} \geqslant \mathrm{S}$. Ce terme a été étudié par RuTter (1971) qui a remarqué une bonne linéarité entre l'eau retenue à la surface des feuilles et le logarithme de l'égoutement :

$$
\mathrm{D}=\mathrm{K}\left(\exp b_{1} \mathrm{C}-1\right)
$$

Les constantes $\mathrm{K}$ et $\mathrm{b}_{\mathrm{n}}$ sont choisies de telle manière que pour $\mathrm{C}=\mathrm{S}$ on $\mathrm{a}$ $\mathrm{D}=0,1 \mathrm{~mm} / \mathrm{h}$. Il ne reste donc plus qu'une seule inconnue à déterminer.

Les valeurs choisies pour la capacité maximale de saturation $S$ sont respectivement de $1,9 \mathrm{~mm}$ pour le hêtre et de $3,9 \mathrm{~mm}$ pour le Douglas.

On définit une évaporation potentielle théorique EP correspondant à la saturation d'une surface plane (PERrier, 1975 I) qui représente le couvert :

avec :

$$
E P=\frac{P^{\prime} R_{x}{ }^{*}+\left(\gamma+\gamma_{1 B}\right) E_{i 1}}{\gamma+\gamma_{1 i}+P^{\prime}}
$$

$$
E_{a}=\frac{L M}{R T_{a}} \cdot \frac{1}{r_{a}} \cdot\left|e_{w}\left(T_{u}\right)-e\right|
$$

Mais l'évaporation d'un couvert entièrement mouillé par l'eau interceptée est inférieure à cette valeur car elle subit un frein supplémentaire dû à la structure du couvert.

En effet nous sommes en présence d'une succession de films d'eau insérés dans l'architecture du couvert. Nous avons donc ajouté à la résistance aérodynamique $r_{\text {ia }}$ (cf. 5.1.) une résistance de structure $r_{* t r}^{*}$ permettant de prendre en compte ce frein à l'évaporation créé par le feuillage (fig. 1). selon le schéma classique de Perrier (1975, II). Ceci revient à considérer qu'une partie de l'arbre participe uniquement à la révaporation de l'eau interceptée et que l'autre partie participe uniquement à la transpiration. Si le feuillage est entièrement saturé en eau alors l'évaporation est égale à :

$$
E_{\mathrm{i}}=\mathrm{E}_{\mathrm{m}: \mathrm{x}}=\frac{\mathrm{EP}}{1+\frac{\gamma^{\prime}+\gamma_{\mathrm{in}}}{\gamma^{\prime}+\gamma_{\mathrm{r}}+\mathrm{P}^{\prime}} \frac{\mathrm{r}_{\mathrm{str}}^{*}}{\mathrm{r}_{\mathrm{a}}}}
$$

Si le couvert n'est pas entièrement saturé en eau $(\mathrm{C}<\mathrm{S})$ 
alors :

$$
E_{i}=\frac{C}{S} E_{\max }
$$

Rappelons que le rapport $\mathrm{C} / \mathrm{S}$ représente ainsi le pourcentage de surface non Iranspirante.

L'évolution du stock d'eau interceptée (équation 4) peut donc se réécrire de la façon suivante dans le cas général :

$$
\frac{d C}{d t}=-\frac{C}{S} E_{\text {ma: }}-K\left(\exp b_{0} C-1\right)+(1-p) P_{i}
$$

Les variations de $\mathrm{C}$ étant faibles nous introduirons dans cette équation la valeur de $E_{i}$ au pas de temps précédent, soit $\frac{\mathrm{C}_{0}}{\mathrm{~S}} \mathrm{E}_{\text {max }}$. on en déduit :

$$
\frac{\mathrm{dC}}{\mathrm{dt}}=-\mathrm{K} \exp \mathrm{b}_{0} \mathrm{C}+\mathrm{Q}
$$

avec :

$$
Q=(1-p) P_{i}-\frac{C_{o}}{S} E_{\max }+K
$$

d'où on en déduit par intégration :

$$
\mathrm{b}\left(\mathrm{C}-\mathrm{C}_{0}\right)=\mathrm{L}_{\mathrm{n}} \mathrm{Q}+\mathrm{b}_{0} \mathrm{QT}-\mathrm{L}_{\mathrm{n}}\left[\mathrm{K} \exp \left(\mathrm{b}_{0} \mathrm{QT}+\mathrm{b}_{0} \mathrm{C}_{0}\right)-\mathrm{K} \exp \mathrm{b}_{0} \mathrm{C}_{\mathrm{0}}+\mathrm{Q}\right]
$$

où le terme $Q$ est considéré, pour l'intégration, comme constant pendant le pas de temps.

\subsection{Gestion de la réserve en eau du sol}

L'équation du bilan hydrique de la tranche de sol participant à la transpiration de l'arbre s'écrit :

$$
\Delta R=R(t+1)-R(t)=p P_{i}+D-(1-C / S) E T R-E C
$$

La réserve utile, $\mathrm{RU}$, valeur maximale de la réserve $\mathrm{R}$ disponible pour les arbres, a été prise égale à $170 \mathrm{~mm}$, valeur de déstockage du sol correspondant au profil de dessèchement maximum (observé à la fin du mois d'août 1976). Les peuplements étudiés sont situés sur un sol brun lessivé marmorisé qui s'est développé sur des marnes liasiques couvertes de limons. La profondeur de sol utile exploitée par le système racinaire des arbres est limité aux 70 premiers centimètres du fait de la présence d'un horizon compact imperméable à cette profondeur, ce qui entraîne l'existence d'une nappe perchée temporaire en hiver ou au début du printemps (Aussenac, 1972). Ce profil pédologique particulier donne à ce site le caractère d'un «lysimètre naturel ". Si l'on table sur une réserve unitaire de $2 \mathrm{~mm} / \mathrm{cm}$, la profondeur 
utile correspond à une réserve utile totale de $140 \mathrm{~mm}$, valeur à laquelle on ajoute $30 \mathrm{~mm}$ correspondant au maximum de sursaturation du sol en présence d'une nappe perchée (Aussenac, comm. pers.), soit en tout $170 \mathrm{~mm}$.

Dans l'équation 13 le terme EC d'écoulement souterrain n'existe que si $\mathrm{R}(\mathrm{t}+1)$ dépasse la valeur $\mathrm{RU}$; $\mathrm{EC}$ est alors égal à $\mathrm{R}(\mathrm{t}+1)-\mathrm{RU}$. Les écoulements latéraux sont négligés.

L'évapotranspiration réelle (correspondant au cas où les feuilles sont sèches) est calculée par la formule suivante, correspondant à un schéma de trois résistances en série (PERRIER, 1975 II et fig. 1).

$$
\operatorname{ETR}=\frac{\mathrm{EP}-\frac{\gamma^{\prime}+\gamma_{\mathrm{l}}}{\mathrm{P}^{\prime}+\gamma+\gamma_{\mathrm{R}}} \cdot \frac{1-\mathrm{U}_{\mathrm{s}}}{\mathrm{U}_{\mathrm{s}}} \cdot \mathrm{h}_{\mathrm{r}} \mathrm{e}_{\mathrm{w}}\left(\mathrm{T}_{\mathrm{d}}\right)}{1+\frac{\gamma^{\prime}+\gamma_{\mathrm{i}}}{\mathrm{P}^{\prime}+\gamma^{\prime}+\gamma_{\mathrm{i}}}\left(\frac{1-\mathrm{U}_{\mathrm{s}}}{\mathrm{U}_{\mathrm{s}}}+\frac{1}{\mathrm{U}_{\mathrm{s}}} \cdot \frac{\mathrm{r}_{\mathrm{str}}+\mathrm{r}_{\mathrm{sto}}}{\mathrm{r}_{\mathrm{a}}}\right)}
$$

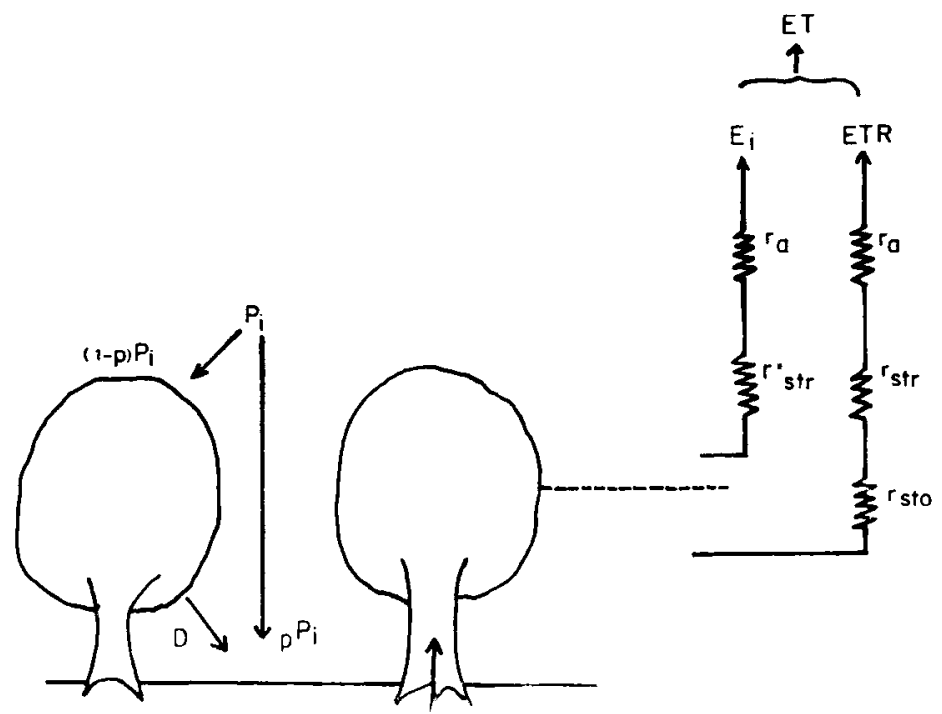

FIG. 1

Modélisation des transferts d'eat et schéma des résistances. Water transfer model and scheme of resistances.

L'humidité relative de surface $U_{*}$ permet de relier le niveau d'évapotranspiration et la disponibilité de l'eau dans le sol (Cholsnel, 1985 b). Celui-ci est schématisé par un système à deux réservoirs. La variable $U_{*}$ est une fonction exponentielle de la profondeur $P$ à laquelle se trouve le niveau de l'eau soit dans le réservoir superficel, soit dans le réservoir profond si le premier est vide. 


$$
U_{s}=\exp \left(-C_{s} \frac{P}{\text { PROF }}\right)
$$

La constante $\mathrm{C}_{\mathrm{s}}$ a été prise égale à sa valeur standard de 0,8 (CHOISNEL, $1985 \mathrm{~b}$ ). PROF est la profondeur utile de sol ; elle correspond à la profondeur exploitée par les racines.

\section{Les données météorologiques}

\subsection{Le fichier climatique utilisé}

Pour résoudre les équations du modèle nous avons besoin de 5 types de données météorologiques : températures de l'air, humidités de l'air, vent, rayonnement et précipitations. Ce modèle a la particularité d'utiliser des données météorologiques de routine disponibles dans toutes les stations synoptiques, à savoir : les températures minimale et maximale journalières, les humidités relatives minimale et maximale, le vent moyen journalier (calculé par la moyenne de 8 observations tri-horaires), la durée d'insolation quotidienne, les cumuls diurnes et nocturnes de précipitations (période de 12 heures) et le code «temps présent».

\subsection{Transformation de ces données}

Pour être compatible avec les hypothèses du bilan d'énergie de surface en particulier la condition de régime quasi-permanent, et afin de réaliser une modélisation physique correcte de l'interception, un pas de temps horaire a été choisi. Il nécessite donc de reconstituer les cycles nycthéméraux des variables météorologiques.

Les températures et les humidités relatives sont estimées heure par heure à l'aide de coefficients horaires valables en France pour chaque saison (CHOISNEL, 1977).

En ce qui concerne le vent, on le considère constant et égal à la moyenne quotidienne. Nous expliquerons plus loin comment à partir de cette donnée mesurée sur un aéroport nous pouvons estimer un coefficient d'échange à l'échelle de la forêt $\left(-\frac{1}{-}\right)$.

Cette approximation est justifiée par le fait que la résistance aérodynamique $\mathbf{r}_{\mathbf{a}} \mathbf{n}$ 'est pas un paramètre crucial du modèle et elle est d'un ordre de grandeur inférieur aux autres résistances. Les flux de rayonnement solaire global et atmosphérique sont estimés à partir de l'insolation et de la tension de vapeur (PERrin DE Brichambaut, 1976).

Pour le calcul de la pluie horaire nous utilisons le code météorologique « Temps présent» (Manuel des codes météorologiques, 1981). Toutes les 3 heures ce code permet de traduire la présence ou non de pluie et son type. S’il indique pluie ou neige la précipitation durera 3 heures. S’il indique averse la précipitation durera 1 heure. 
Il suffira alors de diviser la précipitation totale par le nombre d'heures et d'affecter à celles-ci l'intensité moyenne obtenue. Cette approche approximative ne se justifie que par la nature des données effectivement disponibles dans les fichiers climatologiques. Une pluviométrie véritablement mesurée au niveau horaire permettrait de mieux restituer les épisodes pluvieux.

\section{Les paramètres du modèle}

Le présent modèle utilise le concept de résistance. Examinons successivement les 3 types de résistances.

\subsection{Résistance aérodynamique}

En nous appuyant sur les travaux de SiLversides (1978) nous avons estimé un coefficient d'échange convectif "régional» qui permet de calculer un flux moyen turbulent au-dessus d'une forêt à partir de la mesure du vent au-dessus d'un aéroport (lieu bien dégagé avec un couvert végétal de type gazon). En condition de neutralité thermique le profil vertical de vent est est donnée par une loi logarithmique.

Sur l'aéroport :

$$
V_{a}\left(Z_{a}\right)=\frac{u_{* a+}}{k} \cdot L_{11}\left(\frac{Z_{a}}{Z_{a a}}\right)
$$

Sur la forêt :

$$
V_{f}\left(Z_{f}\right)=\frac{u_{* f}}{k} \cdot L_{n}\left(\frac{Z_{f}-d}{Z_{o f}}\right)
$$

En utilisant la relation empirique de SWINBank (1974) liant la vitesse de frottement $u_{*}$, le vent géostrophique $V_{x}$ et le nombre de Rossby $R_{o}$, et en admettant que le vent géostrophique est le même au-dessus de l'aéroport et au-dessus de la forêt il vient :

$$
\frac{V_{f}}{V_{a 1}}=\left(\frac{Z_{o f}}{Z_{i: a t}}\right)^{0.11 i} \frac{L_{11} \frac{Z_{f}-d}{Z_{0 f}}}{L_{11} \frac{Z_{: 1}}{Z_{0: 3}}}
$$

Nous pouvons ainsi calculer la résistance aérodynamique de la forêt.

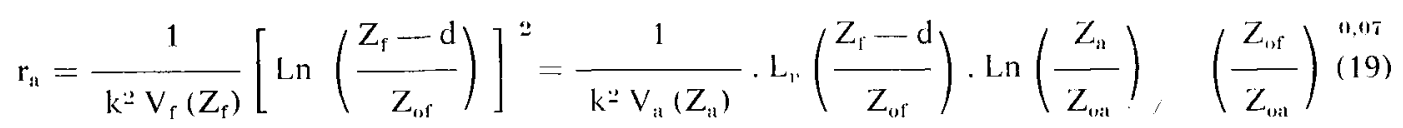

avec : $d=0,75 \mathrm{H}$ et $\mathrm{Z}_{\mathrm{of}}=0,1 \mathrm{H}$ 
Pour améliorer de telles formules il serait nécessaire de disposer de caractéristiques relatives au peuplement (densité et forme), sachant que dans la littérature le rapport $\mathrm{d} / \mathrm{H}$ varie de 0,6 à 0,9 suivant les auteurs.

\subsection{Résistances de structure}

Nous avons été amené à introduire deux résistances de structure différentes, l'une pour l'évaporation de l'eau interceptée, l'autre pour l'eau évapotranspirée.

\subsection{Résistance de structure pour la réévaporation de l'eau interceptée}

Cette résistance permet de tenir compte du fait que nous n'avons pas une surface évaporante unique constituée d'un plan d'eau libre mais un ensemble discontinu de surfaces évaporantes insérées dans le couvert. Elle est donc entièrement dépendante de l'architecture de l'arbre et en particulier de l'indice foliaire. Nous avons supposé qu'elle était une fonction linéaire croissante de celui-ci :

$$
r_{s t r}^{*}=a+b \frac{L A I}{L A I_{\max }}
$$

Le couple de valeurs (a, b) a été choisi de façon à minimiser l'erreur absolue entre interception mesurée et interception calculée au niveau mensuel et annuel. Pour une résistance en $\mathrm{s} / \mathrm{m}$, le meilleur couple est $(20,70)$. Cette résistance diffère de la résistance de structure définie ci-dessous par le fait qu'elle dépend à la fois de la disposition des feuilles et de la façon dont chaque feuille a retenu l'eau, toutes ces surfaces évaporantes n'étant pas forcément saturées en eau.

\subsection{Résistance de structure pour la transpiration}

Perrier (1975) a introduit pour un couvert végétal ayant une certaine extension verticale cette résistance de structure pour tenir compte du fait que l'évaporation ne se produit pas au niveau potentiel théorique maximal. Cette seconde résistance de structure notée $\mathrm{r}_{\text {str }}$ a donc un rôle identique à $\mathrm{r}_{\text {str }}^{*}$ mais elles sont a priori différentes. En effet, dans le cas d'un couvert à la capacité maximale de saturation par interception, la répartition de l'eau diffère peu d'un niveau vertical à l'autre. Par contre le rôle transpiratoire des feuilles n'est pas le même en haut et en bas de la couronne. Ce sont les premières qui transpirent le plus en relation avec la structure des couronnes et le type de feuilles (Aussenac \& DuCREY, 1977). On peut donc supposer que globalement la transpiration de l'arbre subira un frein dû à la structure plus faible dans le cas "transpiration" que dans le cas «interception". C'est ce que nous avons obtenu par ajustement des résultats du modèle aux mesures disponibles, soit :

$$
\mathrm{r}_{\mathrm{str}}=0,5 \mathrm{r}_{\mathrm{str}}^{*}
$$

Cette résistance ne dépend que de la façon dont les feuilles sont réparties dans l'espace. 


\subsection{Résistance stomatique}

L'évolution nycthémérale de l'ouverture et de la fermeture des stomates impose une valeur minimale de la résistance stomatique en l'absence de stress hydrique dans le sol. Nous avons utilisé la formule de Lohammar (cité par Halıdin. 1979; 1980).

$$
r_{s+1 !}=\frac{R_{g}+R_{p g}}{R_{y}}\left(\frac{\alpha+\beta\left[\mathrm{e}_{w}\left(T_{i 1}\right)-e\right]}{L A I}\right)
$$

Les coefficients $\alpha$ et $\beta$ de cette formule ont fait l'objet d'un ajustement d'une part pour les feuillus, d'autre part pour les résineux (cf. tableau 1).

\section{Tableau 1}

Paramètres de la résistance stomatique.

Parameters of stomatal resistance.

\begin{tabular}{|c|c|c|c|}
\hline & $\mathbf{R}_{t}\left(\mathrm{~W} / \mathrm{m}^{2}\right)$ & $u$ & $\beta$ \\
\hline $\begin{array}{l}\text { Feuillu . } \\
\text { Résineux }\end{array}$ & $\begin{array}{l}110 \\
150\end{array}$ & $\begin{array}{l}30 \\
35\end{array}$ & $\begin{array}{l}0,12 \\
0,15\end{array}$ \\
\hline
\end{tabular}

- Les flux de rayonnement sont exprimés en Watt/m².

- Le déficit de saturation est exprimé en Pascals.

- En phase nocturne $(R \mathrm{~g}=0) \mathrm{r}_{\mathrm{st},}=1000 \mathrm{~s} / \mathrm{m}$.

- Pour des feuillus $r_{\text {st }}$ est multiplié dun facteur 4 tant que le LAI n'a pas atteint sa valeur maximale pour tenir compte de la mauvaise efficience respiratoire des premières feuilles.

\subsection{Pourcentage de précipitations atteignant directement le sol}

Ce paramètre $p$ est un facteur de type géométrique estimant les trouées dans le couvert forestier. Pour un feuillage pleinement développé la valeur de 0,25 semble une bonne estimation. Pour le couvert de feuillus ce facteur est une fonction linéaire décroissante de l'indice foliaire.

$$
p=c_{1}+c_{2}\left(\frac{\mathrm{LAI}_{\operatorname{mix}}-\mathrm{LAI}}{\mathrm{LAI}_{\max }}\right)
$$

\subsection{Autres paramètres}

L'albedo des résineux est supposé constant $(0,11)$ tandis qu'il varie suivant la saison pour les feuillus ( 0,12 en hiver, 0,18 en été). Vu l'importance du paramètre 
indice foliaire qui intervient dans la variation au cours du temps des paramètres précédents un paragraphe spécial lui est consacré ci-après.

\section{Indice foliaire}

Les résistances stomatique et de structure ainsi que la répartition des pluies entre le feuillage et le sol dépendent directement de l'indice foliaire. Pour le Douglas nous avons supposé un indice foliaire constant tout au long de l'année (valeur : 6) ; en revanche pour simuler la variation de l'indice foliaire au cours de l'année nous avons adopté pour le hêtre une version simplifiée du modèle phénologique mis au point par NiZINSKI (1986).

Le débourrement nécessite 10 jours consécutifs avec une température moyenne supérieure à $6,5^{\circ} \mathrm{C}$, sachant qu'il ne peut se produire avant le 16 mars. Pour atteindre le stade de feuillaison complète l'arbre requiert une somme de températures

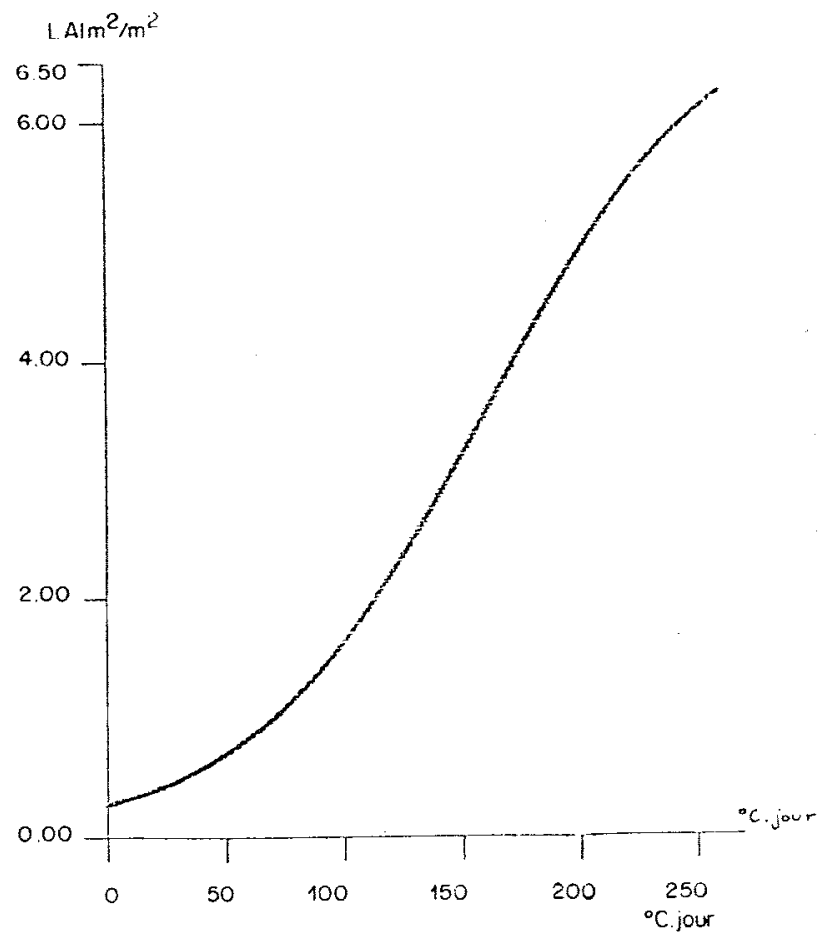

FIG. 2

Evolution de l'indice foliaire du hôtre en fonction des degrés-jours cumulés (base $0^{\circ} \mathrm{C}$ ) à partir de la date de débourrement.

Leaf area index as a function of degree-days (base $0^{\circ} \mathrm{C}$ ) from the date of budding. 
de 259 degrés-jours au-dessus du seuil 0 "C à partir de la date de débourrement. Pendant cette période l'indice foliaire évolue suivant une courbe de type logistique (fig. 2). Pendant la période estivale l'indice foliaire reste constant et il diminue en automne en fonction uniquement de la durée du jour. Il semble que son évolution réelle en automne soit mal prise en compte. Des observations complémentaires seraient nécessaires pour préciser le rôle des facteurs météorologiques, en particulier l'effet du gel ou de vents forts.

\section{Conclusion}

Le modèle présenté ici a pour but de mieux évaluer la consommation en eau d'un couvert forestier. L'introduction des paramètres liés au type de couvert permet de différencier ces consommations suivant les caractéristiques particulières de régulation stomatique (paramètre $r_{\text {sto }}$ ), de densité de plantation et de volume foliaire (paramètres $p$ et L.A.I.). Ainsi est-il possible de mieux cerner l'importance relative de chacun des processus physiques, notamment l'interception, et physiologiques, notamment la phénologie, mis en jeu dans les transferts entre le sol, le couvert forestier et les basses couches de l'atmosphère. 11 permet d'évaluer également le flux global d'évaporation au-dessus d'un massif forestier, flux difficilement mesurable et dont peu de mesures expérimentales sont accessibles, mais dont l'estimation est importante en météorologie (CHOISNEL, 1985 a).

Enfin ce modèle a la particularité d'utiliser comme variables d'entrée des mesures météorologiques standard disponibles en routine dans les stations synoptiques départementales. Il peut également accepter comme variables d'entrée des mesures micrométéorologiques plus fines à condition de réajuster éventuellement certains des paramètres internes du modèle.

Reçu le 19 juin 1985.

Accepté le 27 février 1986.

\section{Remerciements}

Les auteurs remercient MM. Aussenac et Granier de la station de Sylviculture et de Production de l'INRA à Nancy ainsi que MM. Saugier, Pontailler et Nizinssi du Laboratoire d'Ecologie Végétale de la Faculté d'Orsay pour l'aide qu'ils leur ont apportée pour la mise au point de ce modèle.

\section{Summary}

Modelling the total evaporation of a forest canopy

I - Physical principles and model design

This article presents a physical model of the total evaporation and water balance of two types of forest canopies, one of beech (Fagus silvatica L.), the other of Douglas Fir (Psetudotsuga menziesii). It uses exclusively routine measurements at a synoptical meteorological station. In part one of the article the equations governing the calculation of each term of these energy and water balances are detailed as well as how the structural 
and ecophysiologica! parameters, such as the leaf area index, are estimated. Two water balances are calculated : the balance of intercepted water and its rapid re-evaporation, and the soil water balance. The interception sub-model is based on Rutter's model and it uses a Penman-Monteith type formula to estimate the evaporation, in which a structural resistance is introduced and added to the aerodynamic resistance. The tree transpiration model lakes into account three main lactors : the available energy, the stomatal regulation and the soil water status via a double-reservoir system. The evapotranspiration is estimated using Perrier's approach.

The analysis of micrometeorological phenomena such as the interception of rainfall justifies the choice of a hourly time-step for the model. The concept of resistance proved useful in estimating both the re-evaporation of intercepted water and the tree transpiration. Of all the parameters leat area index and its annual variation appear to be a key element, as this parameter is a factor of the distribution of precipitated water, and of the structural and stomatal resistances.

The model has been tested against both interception measurements and weekly neutron measurements of the soil water status at Nancy's Forest Research Centre. model.

Key words: Evaporation, interception, leaf area index, water balance, meteorology,

\section{Références bibliographiques}

Aussenac G., 1968. Interception des précipitations par le couvert forestier. Ann. Sci. For., 25 (3), $135-156$.

Aussenac G., 1972. Etude de l'évapotranspiration réelle de quatre peuplements forestiers dans l'Est de la France. Ann. Sci. For., 29 (3), 369-389.

Aussenac G., 1975. Couverts forestiers et facteurs du climat : leurs interactions, conséquences écophysiologiques chez quelques résineux. Thèse de Docteur es Sciences Naturelles. Nancy, 9 juin 1975, $243 \mathrm{p}$.

Aussenac G., Ducrey M., 1977. Etude bioclimatique d'une futaie feuillue de l'Est de la France. I. Analyse des profils microclimatiques et des caractéristiques anatomiques et morphologiques de lappareil foliaire. Ann. Sci. For., 34 (4), 265-284.

Aussenac G., Granier A., 1979. Etude bioclimatique d'une futaie feuillue (Fagus silvaica 1. et Quercus sessiliflora Salisb.) de l'Est de la France. II. Etude de l'humidité du sol et de l'évapotranspiration réelle. Ann. Sci. For., 36 (4), 265-280.

Aussenac C., Boulangeat C., 1980. Interception des précipitations el évapotranspiration réelle dans les peuplements de feuillus (Fagus silvatica L.) et de résineux (Pseudotsuga menziesii (Mirb) Franco). Ann. Sci. For., 37 (2), 91-107.

AVERY C.A., Fritschen L.J., 1971. Hydrologic and energy budgets of stocked and non stocked Douglas-fir sites as calculated by meteorological methods. University of Washington, College of Forest Resources. Research Completion Report, $131 \mathrm{p}$.

Chassagneux P., 1984. Etude et modélisation de l'évaporation globale et du bilan hydrique d'un couvert forestier. Rapport de stage de recherche de l'Ecole Nationale de la Météorologie, Subdivision d'Agrométéorologie, Paris, 84 p.

Choisnel E., 1977. Le bilan d'énergie et le bilan hydrique du sol. La Météorologie, numéro spécial «Evapotranspiration », 6 (11), 103-159.

Choisnel E., 1985 a. Cycle de l'eau dans l'atmosphère et rôle des zones forestières. La Météorologie, 7 (9), 37-50.

Choisnel E., 1985 b. Un modèle agrométéorologique opérationnel de bilan hydrique utilisant les données climatiques. In : Les besoins en eau des cultures. Paris, ed. INRA, $115-132$.

Halldin S., Gripp H., Perttu K., 1979. Model for energy exchange of a pine forest canopy. International Society for Ecological Modelling, Copenhagen. In : Comparison of forest water and energy exchange models. S. Halldin ed., Elsevier, Amsterdam, 59-75. 
Lohammar T., larsson S., IINDFR S., FAIK S.O., 1980. FAST - simulation models of gaseous exchange in Scots pincs. In: Stracture and function of Northern coniferous forests. An ecosystem study. Ecol. Bull., 32, Stockholm, 505-527.

MÉTEOROLOGIE NATIONAIE, 1981. Manuel des coles météoroloziques. Direction de la Météorologie, Boulogne, édition de 1981.

Nizinski G., 1986. La modélisation da bilan de l'eau d'une futaie de chèné en forít de Fontainebleau. Thèse de Docteur-Ingénicur, Université dorsay, $101 \mathrm{p}$.

Plorier A., 1975. Etude physique de l'évapotranspiration dans les conditions naturelles. 1. Evaporation et bilan d'énergie des surfaces naturelles. Anu. Agron., 26 (1), 1-18. II. Expressions et paramètres donnant l'évapotranspiration réelle d'unc surface « mince ». Ann. Agron., 26 (2), 105-123. IlI. Evapotranspiration réelle el potentielle des couverts végétaux. Anh. Agron., 26 (3), 229-243.

Pirrin de Brichambaut C., 1976. Météorologie el énergie : lévaluation du «gisement solaire 》. La Météorologie, 6 (5), 129-158.

Ruther A.J., Kershaw K.A., Robbins P.C., Morgon A.J., 1971. A predictive model of rainfall interception in forests. I. Derivation of the model from observations in a plantation of Corsican pine. Agric. Meteorol.. 9, 367-384.

Sil.versides R.H., 1978. Forest and airport wind speeds. Atmosphere - Ocear, 16 (3), $293-299$.

Swindank W.C., 1974. The geostrophic drag coefficient. Bolndary Layer Meteor, 7, $125-127$.

\section{Liste des symboles utilisés}

a. b : coefficients de la résistance de structure

$b_{4} \quad$ : coefficient de la formule de l'égouttement

$c_{1}, c_{2}$ : coefficients de la formule donnant $\mathrm{p}$

C : quantité d'eau sur lé feuillage $(\mathrm{mm})$

$\mathrm{C}_{\text {" }} \quad$ : valeur de $\mathrm{C}$ au début du pas de temps

$\mathrm{C}_{*} \quad$ : constante dans la formule donnani $\mathrm{U}_{*}$

d : hauteur de déplacement de la forêt

D : égouttement ( $\mathrm{mm}$ )

e. : tension de vapeur (Pa)

$c_{w} \quad$ : tension de vapeur saturante $(P a)$

$\mathrm{E}_{\mathrm{a}} \quad$ : pouvoir évaporant de l'air

EC : écoulement $(\mathrm{mm})$

$E_{i} \quad$ : évaporation de l'eau interceptéc

$\mathrm{E}_{\text {max }}$ : évaporation de l'cau interceptée dans le cas où l'arbre est totalement mouillé $(\mathrm{mm})$

EP : évaporation potentielle théorique

ETR : évapotranspiration réelle dans le cas où les feuilles sont sèches

ET : évapotranspiration réelle totale

$8 \quad$ : émissivité infrarouge de grande longueur d'onde

$\mathbf{h}_{\text {. }} \quad$ : coefficient d'échange par évaporation, proportionnel à $1 / \mathrm{ra}$

$\mathrm{H}$ : hauteur de la forêt

$k \quad$ : constante de VON KARMAN

K : coefficient de la formule de l'égouttement

L : chaleur latente de vaporisation 
LAI : indice foliaire $\left(\mathrm{m}^{2} / \mathrm{m}^{2}\right)$

$\mathrm{LAI}_{\max }$ : indice foliaire maximal

M : masse molaire de l'eau

$\mathrm{p} \quad$ : pourcentage de précipitations atteignant directement le sol

$\mathrm{P} \quad$ : profondeur de l'eau dans le réservoir analngique

$\mathrm{P}^{\prime} \quad: \mathrm{d}_{\mathrm{i} w} / \mathrm{dT}$

$\mathrm{P}_{\mathrm{i}} \quad$ : pluie incidente

PROF : profondeur utile du sol

$\mathrm{R} \quad$ : constante des gaz parfaits

$R(t) \quad$ : réserve au temps $(t)$

$r_{i t} \quad:$ résistance aérodynamique $(\mathrm{s} / \mathrm{m})$

$\mathrm{R}_{\mathrm{g}} \quad$ : rayonnement solaire global $\left(\mathrm{W} / \mathrm{m}^{2}\right)$

$\mathrm{R}_{\mathrm{g} o}, \alpha, \beta$ : coefficients de la formule donnant $r_{\mathrm{sto}}$

$\mathrm{RN}^{*}$ : rayonnement net climatique

$\mathrm{R}_{0} \quad$ : nombre de Rossby

$\mathrm{r}_{\mathrm{sto}} \quad$ : résistance stomatique $(\mathrm{s} / \mathrm{m})$

$\mathrm{r}_{\mathrm{str}}^{*} \quad:$ résistance de structure pour leau interceptée $(\mathrm{s} / \mathrm{m})$

$\mathbf{r}_{\mathrm{str}} \quad$ : résistance de structure pour la transpiration

RU : réserve utile

$\mathrm{S} \quad$ : capacité maximale de saturation

$\sigma \quad$ : constante de Stefan-Boltzmann

$\mathrm{t} \quad:$ le temps

$\mathrm{T}_{\mathrm{a}} \quad$ : température de l'air

$\mathrm{T}_{\mathrm{d}}$ : température du point de rosée

$\mathrm{T}_{\mathrm{s}} \quad$ : température de surface du couvert

$\mathrm{U}_{\mathrm{s}} \quad$ : humidité relative de surface

$u_{*} \quad:$ vitesse de frottement

$\mathrm{V}_{\mathrm{a}}\left(\mathrm{z}_{\mathrm{a}}\right)$ : vent mesuré à la hauteur $\mathrm{z}_{\mathrm{a}}(10 \mathrm{~m})$ à l'aéroport

$V_{f}\left(z_{f}\right)$ : vent estimé à la hauteur $z_{f}(10 \mathrm{~m})$ au-dessus de la forêt

$V_{\mathrm{g}} \quad$ : vent géostrophique

$Z_{\text {oa }} \quad:$ hauteur de rugosité de l'aéroport $(1 \mathrm{~cm})$

$\mathrm{Z}_{\mathrm{of}} \quad$ : hauteur de rugosité de la forêt

$\gamma \quad:$ coefficient psychrométrique

$\gamma_{\mathrm{r}}$ : coefficient correcteur de $\gamma$ (linéarisation du flux radiatif)

$B_{\mathrm{k}} \quad$ : flux de conduction dans le sol 